\title{
Inhibition of LncRNA-HRIM Increases Cell Viability by Regulating Autophagy Levels During Hypoxia/Reoxygenation in Myocytes
}

\author{
Zhouqing Huang Bozhi Ye Zhengxian Wang Jibo Han Lu Lin Peiren Shan \\ Xueli Cai Weijian Huang
}

Department of Cardiology, the key lab of Cardiovascular disease of Wenzhou, the First Affiliated Hospital of WenZhou Medical University, WenZhou, ZheJiang, P.R. China

\section{Key Words}

Long noncoding RNA • Autophagy • Myocardial Cell $\bullet$ Ischemia/Reperfusion Injury • SiRNA

\begin{abstract}
Backgrund/Aims: Ischemia reperfusion (I/R) promotes the severity of cardiomyocyte injury. Long noncoding RNAs (LncRNAs) are key regulators in cardiovascular diseases. However, the association between LncRNAs and myocardial I/R injury has not been thoroughly characterized to date. We attempted to clarify the potential biological role of a LncRNA (E230034O05Rik), which we named hypoxia/reoxygenation (H/R) injury-related factor in myocytes (HRIM), by investigating the differential expression of LncRNAs between groups of myocytes exposed to either a normal level of oxygen or to H/R. Methods: Microarray analysis was used to determine analyze the global differential expression of LncRNAs in H9c2 myocytes exposed either to a normal level of oxygen or to H/R. Target LncRNA levels were further verified in vitro and ex vivo by real-time polymerase chain reaction ( $(\mathrm{PCCR})$. Cell viability was analyzed using the Cell Counting Kit- 8 assay. Autophagy levels were confirmed by Western blotting, transmission electron microscopy, and autophagic double-labeled (mRFP-GFP-LC3) adenovirus analyses. Results: Gene expression profiling revealed that 797 LncRNAs and 1898 mRNAs were differentially expressed in the $\mathrm{H} / \mathrm{R}$ group compared with the normal oxygen group. Among these LncRNAs and mRNAs, 6 upregulated LncRNAs and 2 downregulated LncRNAs in the H/R group were selected and further validated by $q P C R$ in vitro and ex vivo. Additionally, LncRNAHRIM was inhibited by specific siRNAs in H9c2 myocytes exposed to H/R. The inhibition of LncRNA-HRIM by siRNA prevented cell death by suppressing excessive autophagic activity in myocytes, This finding suggests a detrimental role of LnCRNA-HRIM in the regulation of I/R injury. Conclusions: LncRNAs are involved in H/R injury of H9c2 myocytes. Inhibition of LncRNA-HRIM increased cell viability by reducing autophagy in myocytes during $H / R$.
\end{abstract}

Zhouqing Huang and Bozhi Ye contributed equally to this work.

Weijian Huang and Zhouqing Huang 


\section{Cellular Physiology Cell Physiol Biochem 2018;46:1341-1351 \begin{tabular}{ll|l} 
and Biochemistry Published online: April 23, 2018 & $\begin{array}{l}\text { (C) } 2018 \text { The Author(s). Published by S. Karger AG, Basel } \\
\text { www.karger.com/cpb }\end{array}$
\end{tabular} \\ Huang et al.: LncRNA-HRIM Regulated Autophagy}

\section{Introduction}

Acute myocardial infarction (MI) is a common cause of death that results from atherosclerotic coronary heart disease (CHD). Reperfusion therapies, including primary percutaneous coronary intervention or thrombolytic therapy, benefit CHD patients [1]. However, such therapies may cause myocardial ischemia/reperfusion (I/R) injury. Therefore, reduction of reperfusion injury is an important topic in the treatment of MI. However, the pathogenesis of I/R injury has not been thoroughly elucidated to date.

Recent research reported that autophagy is activated during the I/R period and likely contributes to cell death by excessively degrading essential proteins and organelles [2]. During the reperfusion period, inhibition of autophagy with berberine [3] or curcumin [4] significantly mitigates I/R injury, which reduces the scope of MI and improves cardiac function. These findings suggest that excessive autophagy plays an adverse role during I/R period injury. Hence, appropriate regulation of autophagy is beneficial for I/R injury treatment.

A growing body of research revealed that noncoding RNAs are involved in various biological functions in the regulation of pathophysiological conditions, such as cancer [5], autoimmune diseases [6], and inflammation-related illnesses [7]. Moreover, long noncoding RNAs (LncRNAs) and endogenous RNAs that contain over 200 nucleotides can combine with biological molecules (e.g., mRNAs, miRNAs, or proteins) and influence key proteins or signaling pathways in different pathophysiological processes. The pathogenesis of certain diseases may be related to abnormal LncRNA expression [8]. For example, the aberrant expression of LncRNAs, such as Mhrt, is associated with the pathogenesis of ischemic heart failure $[8,9]$. However, the role of LncRNAs in cardiac I/R injury remains unclear. In the present study, we investigated the differential expression of LncRNAs in myocytes during I/R using in vitro and ex vivo models. Furthermore, we determined the effect of LncRNA E230034005Rik on the regulation of autophagy in myocytes. We designated LncRNA E230034005Rik as hypoxia/reoxygenation (H/R) injury-related factor in myocytes (HRIM).

\section{Materials and Methods}

\section{Reagents}

Dulbecco's Modified Eagle Medium (DMEM), fetal bovine serum (FBS), and penicillin/streptomycin (pen/strep, 10, $000 \mathrm{U} / \mathrm{ml}$ each) were purchased from GIBCO (Life Technologies, Shanghai China). AntiGAPDH and LC3B antibodies were procured from Cell Signaling Technology (Danvers, MA USA). Goat anti-rabbit secondary antibodies (Catalog A-21109) used in Western blot were purchased from Invitrogen (Carlsbad, CA USA).

\section{Animals}

Male Sprague Dawley rats were obtained from the Shanghai Animal Center Laboratory (Shanghai, China). Animal procedures were performed in accordance with the instructions outlined in the Guidelines for the Care and Use of Laboratory Animals (US National Institutes of Health). Animal care and experimental protocols were approved by the Committee on Animal Care of Wenzhou Medical University (Wenzhou, Zhejiang, China).

\section{Experimental protocol and induction of $I / R$}

Ten Sprague Dawley rats (aged, 8 weeks to 12 weeks, body weight280 g to $320 \mathrm{~g}$ ) on a standard diet were randomly divided into two groups: the sham group (sham, $n=5)$ and the I/R group $(\mathrm{I} / \mathrm{R}, n=5)$. Surgery was performed as described previously [10]. Briefly, rats were anesthetized with an intraperitoneal injection of $10 \%$ chloral hydrate $(300 \mu \mathrm{l} / \mathrm{kg})$. Each rat was completely sedated. The chest was opened via thoracotomy and ribs were removed. The pericardium was ruptured and the aorta was exposed. A steel cannula was later placed into the aorta and secured with a suture. The heart was immediately perfused with Krebs-Henseleit bicarbonate buffer at a constant pressure of $60 \mathrm{mmHg}$ to $70 \mathrm{mmHg}$ at $37^{\circ} \mathrm{C}$. Thirty minutes 


\section{Cellular Physiology Cell Physiol Biochem 2018;46:1341-1351

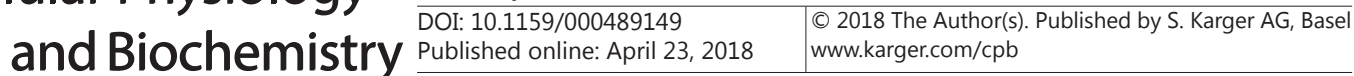 \\ Huang et al.: LncRNA-HRIM Regulated Autophagy}

before the operation, the buffer was bubbled with $95 \% \mathrm{O}_{2}$ and $5 \% \mathrm{CO}_{2}$ to attain a $\mathrm{pH}$ of 7.4 . The heart was quickly excised and transferred into a Langendorff apparatus while perfusion continued [11]. All hearts were perfused for 20 min before induction of ischemia to attain equilibrated coronary perfusion pressure (CPP). Hearts were subsequently subjected to $30 \mathrm{~min}$ of no-flow global ischemia, followed by $120 \mathrm{~min}$ of reperfusion [11]. Hearts that showed arrhythmia during the equilibration, had sustained reduction in CPP, or had flow less than $8 \mathrm{ml} / \mathrm{min}$ were excluded from the study. ECG, CPP, and perfusion flow parameters were monitored before the induction of ischemia and during reperfusion with a Power lab recorder (AD Instruments, Australia). The successful induction of ischemia was determined by ST segment elevation on an electrocardiogram.

\section{Hypoxia/reoxygenation with H9c2 myocytes}

The rat myocardium-derived H9c2 cardiac myoblast cell line was purchased from American Type Culture Collection (Rockville, MD, USA). Cells were maintained in DMEM containing 4, $500 \mathrm{mg} / \mathrm{L}$ glucose and supplemented with 10\% ( $/ / v$ ) FBS, $10 \mathrm{mM}$ HEPES (Sigma), and $1 \%$ pen/strep solution at $37{ }^{\circ} \mathrm{C}$ in a humidified $5 \% \mathrm{CO}_{2}$ incubator. To simulate I/R injury, H/R was induced in H9c2 myocytes. The cells were cultured in FBS and glucose-deprived DMEM, and were first exposed to hypoxic conditions $\left(1 \% \mathrm{O}_{2}, 5 \% \mathrm{CO}_{2}\right.$, and $94 \% \mathrm{~N}_{2}$ ) for $1 \mathrm{~h}$ and normoxic conditions for $3 \mathrm{~h}$. Meanwhile, the cells in the control group (without FBS and glucose-deprived DMEM) were maintained under normoxic conditions $\left(21 \% \mathrm{O}_{2}, 5 \% \mathrm{CO}_{2}\right.$, and $\left.74 \% \mathrm{~N}_{2}\right)$ for $4 \mathrm{~h}$.

\section{Microarray and computational analysis}

Total RNA was extracted from each sample (3 normal oxygen groups and $3 \mathrm{H} / \mathrm{R}$ treatment groups), amplified, and transcribed into fluorescent cRNA with the Quick Amp Labeling kit (Agilent Technologies, Palo Alto, CA,USA). Labeled samples were hybridized to the rat LncRNA Array ( 4 x 44K, ArrayStar, Rockville, MD,USA), which detected the expression profiles of rat genome-wide protein-coding transcripts (KangChen Bio-tech, Shanghai, China). The arrays were washed and then scanned using the Agilent Scanner G2505B. Acquired array images were analyzed with Agilent Feature Extraction Software (version 10.5.1.1). Quantile normalization and subsequent data processing were performed with the GeneSpringGX v11.0 software package (Agilent Technologies). The statistically significant differential expression of LncRNAs was identified using the criteria of a fold change $>1.5$ and $p<0.05$.

\section{Knockdown of LncRNA E230034005Rik in H9c2 myocytes}

To knockdown E230034005Rik in H9c2 myocytes, H9c2 myocytes were transfected with siRNAs against rat E230034005Rik (full length rat E230034005Rik sequence is available at http://www.ncbi. nlm.nih.gov/nuccore/NR_002154). The siRNAs and negative control (NC) siRNA were synthesized by RiboBio (Guangzhou, China). H9c2 myocytes were randomly divided into four groups: the normal oxygen group (control group), the H/R group, the H/R+NC siRNA group and the H/R+siRNA group. The H/R+NC siRNA group was the control for the $\mathrm{H} / \mathrm{R}+$ siRNA group to eliminate any effects of substance siRNA. H9c2 myocytes were transfected with siRNAs for $48 \mathrm{~h}$ in accordance with the manufacturer's instructions and later subjected to $\mathrm{H} / \mathrm{R}$ treatment as described above.

\section{Gene coexpression network construction}

Gene coexpression networks were visually represented to identify interactions among genes. The method was performed as described by Yang [12]. Gene coexpression networks were assembled based on the normalized signal intensity of specific expressed genes. Pearson's correlation coefficients were applied to calculate gene pair correlations on the basis of gene expression. To ensure visual representation, only the strongest correlations ( 0.99 or greater) were included in the renderings. Each gene corresponded to a node in the gene coexpression network. Two genes connected by an edge indicated a strong correlation (i.e., either positive or negative). Within the network, the node with more edges signified that the gene had more coexpressed genes. Moreover, these genes would be considered in our follow-up studies.

RNA isolation, cDNA synthesis, and TaqMan real-time PCR

H9c2 myocytes were cultured in 12-well plates under similar treatments or in an isolated heart exposed to I/R as mentioned above. mRNA levels were analyzed using SYBR Green reagent kits with gene 


\section{Cellular Physiology Cell Physiol Biochem 2018;46:1341-1351

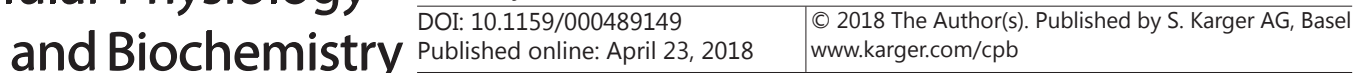 \\ Huang et al.: LncRNA-HRIM Regulated Autophagy}

specific primers on Applied Biosystems 7300 Real-Time PCR system (USA), following the manufacturer's instructions. Amplification conditions were $94^{\circ} \mathrm{C}$ for $7 \mathrm{~min}$, followed by 40 cycles of $95^{\circ} \mathrm{C}$ for $15 \mathrm{~s}$, and 60 ${ }^{\circ} \mathrm{C}$ for $30 \mathrm{~s}$. All primer sequences for RT-PCR assays are listed in Table S1. All experiments were performed in triplicate and all samples were normalized with GAPDH.

Assessment of cell viability with a CCK-8 assay

Cell viability was assessed with the Cell Counting Kit-8 (CCK-8, Beyotime Biotech, Jiangsu, China) in accordance with the manufacturer's instructions. The kit contained a water-soluble tetrazolium salt (WST8) and a substrate of mitochondrial succinate dehydrogenase that converts WST-8 into orange-colored formazan. The activities of these enzymes are proportional to those in living cells. H9c2 myocytes $\left(5 \times 10^{3}\right)$ well) were seeded in 96-well plates and subjected to both control and H/R culture conditions. At the end of each treatment, the culture medium was replaced with $100 \mu \mathrm{L}$ of CCK-8 solution containing $90 \mu \mathrm{L}$ of serumfree DMEM and $10 \mu \mathrm{L}$ of CCK-8 reagent. Absorbance at $450 \mathrm{~nm}$ was measured in each well by visualization of color intensity development.

\section{Transmission electron microscopy}

H9c2 myocytes were cultured in P60 dishes $\left(\sim 1 \times 10^{6}\right)$ under similar treatments as mentioned above. At the end of each experiment, cells were washed with $0.1 \mathrm{M}$ cacodylate buffer and fixed in $2.5 \%$ glutaraldehyde containing $0.1 \mathrm{M}$ cacodylate buffer for $1 \mathrm{~h}$. Cells were scraped off of the plates, collected in Eppendorf tubes and fixed with 1\% osmium tetroxide. Cells were dehydrated in a gradient series of ethanol solutions (from 50\% to 100\%) in Eppendorf tubes and then embedded in epoxy resin. Ultrathin $(\sim 70 \mathrm{~nm})$ sections were cut and placed on grids. The sections were double-stained with uranyl acetate and lead citrate prior to examination under an electron microscope (JEOL, JEM-1230, Japan).

\section{Protein extraction and Western blot analysis}

Cells were lysed with RIPA buffer on ice and then centrifuged at $12,000 \mathrm{rpm}$ for $10 \mathrm{~min}$ at $4{ }^{\circ} \mathrm{C}$. Up to $40 \mu \mathrm{g}$ of total protein from each sample was loaded for SDS-PAGE separation and later transferred onto PVDF membranes as previously described [13]. The PVDF membranes were blocked in 5\% dry milk/TBST and subsequently probed with primary antibodies, including rabbit IgG anti-LC3, at a 1:1000 dilution in 5\% dry milk/TBST or anti-GAPDH at a 1:5000 dilution. The secondary antibody was a goat anti-rabbit labeled with Alexa Fluor 680 (1:2000 dilution). The antibody-labeled membranes were scanned for far-red signals by an Odyssey Imaging System (LI-COR, NE, USA). Data were analyzed with Quantity One Software (Bio-Rad, CA, USA). In addition, protein expression levels were quantified by relative densitometry with actin as the loading control.

\section{Statistical analysis}

All experiments were repeated at least three times and the data are presented as the mean \pm SD. Data were analyzed by one-way ANOVA. Post-hoc analyses, including the Student-Newman-Keuls method and Dunnett's test, were performed using SPSS 18 software (SPSS Inc., Chicago, IL). The statistical significance was defined as $p<0.05$.

\section{Results}

LncRNA expression profiles of rat H9c2 myocytes after $H / R$ treatment

Microarray analysis was performed on three normal oxygen groups and three $H / R$ groups to investigate the differential expression of LncRNAs. The microarray analysis was conducted by KangChen Bio-tech (Shanghai, China). Compared with the control group, the H/R treatment group had 309 upregulated LncRNAs and 488 downregulated LncRNAs with a $>1.5$-fold change and $p<0.05$ (for all online suppl. material, see www.karger.com/doi/ 10.1159/000489149, File S1). Additionally, the microarray data have been deposited in the public domain (GEO number: GSE103731). Moreover, we constructed heat maps and performed hierarchical clustering of LncRNA (Fig. 1A) and mRNA (Fig. 1B) expression ratios (log2 scale), and compared H/R-treated H9c2 myocytes and myocytes that had normal oxygen treatment. 


\section{Cellular Physiology Cell Physiol Biochem 2018:46:1341-1351 and Biochemistry \begin{tabular}{c|c} 
DOI: 10.1159/000489149 & $\begin{array}{l}\text { O 2018 The Author(s). Published by S. Karger AG, Basel } \\
\text { www.karger.com/cpb }\end{array}$
\end{tabular}

Gene ontological (GO) and pathway analyses

To further reveal the potential roles of LncRNAs in $\mathrm{H} / \mathrm{R}$ injury, GO analysis was performed to annotate transcripts with terms related to biological processes, cellular component, and molecular function. The 1898 differentially expressed coding genes were analyzed using GO annotations (see online suppl. material, Suppl. Fig. S1). Additionally, module pathway enrichment was analyzed using Kyoto Encyclopedia of Genes and Genomes (KEGG) to confirm potential module function. A total of 1898 coding genes were identified by ANOVA ( $p<$ $0.05)$. KEGG pathway analysis revealed that the majority of LncRNA target genes were associated with sterol biosynthesis and DNA replication pathways (see online suppl. material, Suppl. Fig. S1). Candidate genes for subsequent experiments were selected based on the information yielded by the GO and KEGG pathway analyses.

Gene coexpression network construction

To reveal the potential mechanism for LncRNAs in H/R injury, the 797 LncRNAs and 1898 coding mRNAs were clustered into phenotypically relevant coexpression modules on the basis of LncRNA and gene coexpression networks, where $R>0.99$. The coexpression networks for eight highly dysregulated LncRNAs are shown in Fig. 1C. The analysis demonstrated that E230034005Rik was coexpressed with coding RNAs (mRNAs) of Zdhhc7, Ptgis, Krt23 and Phactr1, which are linked to the regulation of hypoxia/reoxygenation (H/R) injury in myocytes (Fig. 1C). We confirmed by qPCR that the expression levels of Zdhhc7, Ptgis, Krt23 and Phactr1 were increased in the H/R group compared with the control group. This suggests that Zdhhc7, Ptgis, Krt23 and Phactr1 are coexpressed with E230034005Rik during H/R injury (see online suppl. material, Suppl. Fig. S2).

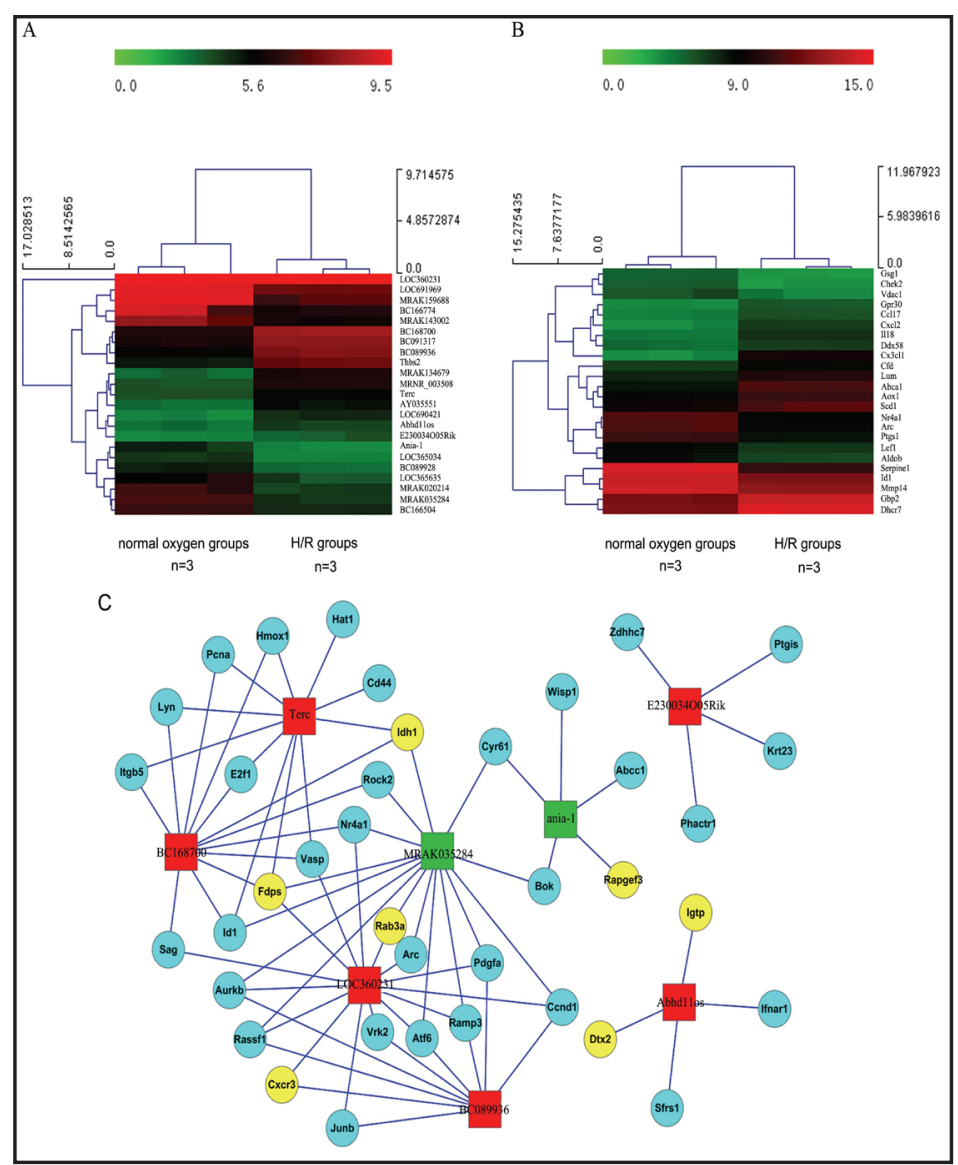

Fig. 1. Differential expression of lncRNAs and mRNAs in H/R groups compared with normoxic groups in H9c2 myocytes. Heat maps and hierarchical clustering of expression ratios (log2 scale) of lncRNAs (A) and mRNAs (B) in H9c2 myocytes under H/R compared with normal oxygen treatment. "Red" denotes a high relative expression in H/R groups $(n=3)$ compared with normal oxygen groups $(n=3)$, whereas "blue" denotes a low relative expression in H/R groups ( $\mathrm{n}=$ 3 ) compared with normal oxygen groups $(n=3)$. (C) The sub-network of 8 highly-dysregulated lncRNAs that were hubbed in the global network and co-expressed with 38 coding mRNAs. Genes colored red are downregulated lncRNAs. Genes colored green are downregulated IncRNAs. Upregulated mRNAs are colored yellow and downregulated mRNAs are colored cyan. 
Validation of misregulated LncRNAs and mRNAs in $H / R$ and $I / R$ treatment

To validate the accuracy of the LncRNA profiles generated by microarray analysis and the success of the H/R and I/R treatment models, we further confirmed the expression levels of 8 LncRNAs and the mRNA expression levels of nppa, bax, bcl-2 and caspase-3 with qPCR analysis. As expected, the expression levels of BC089936, BC168700, Terc, E230034005Rik, Abhd11os, LOC360231, nppa, bax and caspase-3 were significantly increased in the H/R group, whereas the expression levels of ania-1, MRAK035284 and bcl-2 were decreased in the H/R group (Fig. 2A and see online suppl. material, Suppl. Fig. S2). Similar results were observed in Sprague Dawley rats: the expression levels of 6 LncRNAs increased in the I/R group, and the expression levels of 2 LncRNAs decreased in the I/R group (Fig. 2B).

It was difficult to choose a candidate LncRNA for subsequent research based on the large chip data. We selected LncRNA-HRIM for further investigation for the following reasons: (i) LncRNA E230034005Rik was significantly upregulated in the H/R or I/R group compared with the control group, (ii) E230034005Rik is a validated LncRNA and not a pseudogene, and (iii) Clic1, the contiguous gene of E230034005Rik, is related to autophagy, which plays an important role in I/R injury. Furthermore, LncRNA-HRIM is co-expressed with Zdhhc7, Ptgis, Krt23 and Phactr1 mRNAs, which may be linked to the regulation of hypoxia/ reoxygenation (H/R) injury in myocytes. Our results suggest that LncRNA E230034005Rik may be the potential LncRNA that is important for the regulation of hypoxia/reoxygenation $(\mathrm{H} / \mathrm{R})$ injury in myocytes.

\section{Genetic characteristics of LncRNA-HRIM}

LncRNA-HRIM is a long non-coding RNA that is $1470 \mathrm{bp}$ in size. LncRNA-HRIM is located on $20 \mathrm{p} 12$ and has three exons. Its potential regulatory gene is clic1, which has been reported to stimulate cell growth both in vitro and in vivo [14]. We performed RACE and confirmed that LncRNA-HRIM has polyA tail (see online suppl. material, File S2). Furthermore, additional experiments confirmed that the LncRNA-HRIM is expressed in various tissues in rat (see online suppl. material, Suppl. Fig. S2), while, there are low levels of LncRNA-HRIM in human cell samples such as HepG2 and HUVEC, This finding suggests that LncRNA-HRIM has a lack of sequence conservation between species.

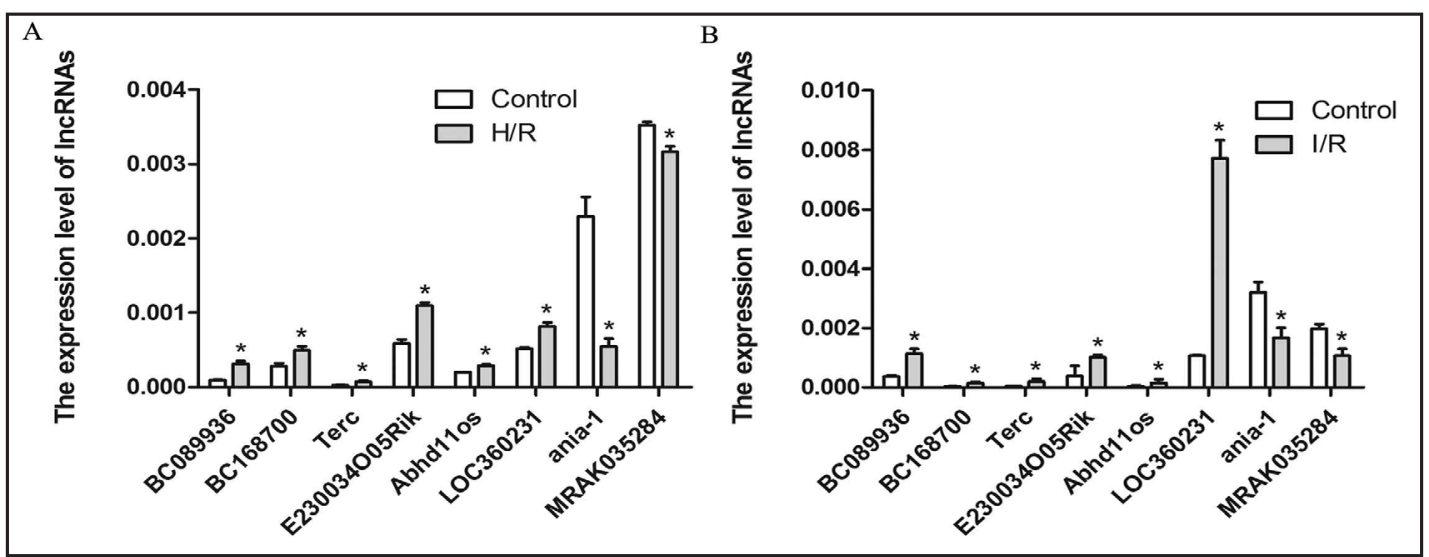

Fig. 2. Quantitative real-time PCR validation of 8 differentially expressed lncRNAs and known H/R-regulated protein-coding genes. (A). The 6 upregulated IncRNAs (BC089936, BC168700, Terc, E230034005Rik, Abhd11os, and LOC360231), 2 downregulated IncRNAs (AF030086, MRAK035284) and I/R-regulated protein-coding gene in the H/R group compared with the normal control in vitro, GAPDH was designated an endogenous reference gene. (B) The 6 upregulated lncRNAs (BC089936, BC168700, Terc, E230034005Rik, Abhd11os, and LOC360231), 2 downregulated IncRNAs (AF030086, MRAK035284) and I/R-regulated protein-coding gene in the I/R group compared with the normal control group ex vivo. GAPDH was designated an endogenous reference gene. The lncRNA unit represents the relative expression of the lncRNA compared to GAPDH. Data (mean \pm SD) were obtained from three independent experiments $(n=5)$. ${ }^{*} \mathrm{P}<0.01$ compared with the control group.

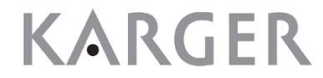




\section{Cellular Physiology Cell Physiol Biochem 2018;46:1341-1351 \begin{tabular}{l|l} 
and Biochemistry Published onlIne: AprII 23, 2018 & $\begin{array}{l}\text { (c) } 2018 \text { The Author(s). Published by S. Karger AG, Basel } \\
\text { www.karger.com/cpb }\end{array}$ \\
\hline
\end{tabular}

\section{Silencing LncRNA-HRIM attenuated H/R injury in H9c2 myocytes}

To evaluate the effects of LncRNA-HRIM on H/R injury, three different siRNAs (designated as siRNA-1, siRNA-2, and siRNA-3) were transfected against LncRNA-HRIM in H9c2 myocytes. As depicted in Fig. 3A, all three siRNAs knocked down the expression of LncRNA-HRIM and the inhibitory effect of siRNA-1 was the most significant. The sequences for siRNA-1 is shown (see online suppl. material) in Suppl. Table S1. Compared with cells in the control group, depletion of LncRNA-HRIM by siRNA-1 increased cell viability in H9c2 myocytes during H/R injury. By contrast, the si-LncRNA-control treatment did not affect cell viability (Fig. 3B). These data suggested that inhibition of LncRNA-HRIM can increase cell survival of H9c2 myocytes during the H/R period.

\section{LncRNA-HRIM regulates autophagy in $H 9 c 2$ myocytes during $H / R$}

Autophagy plays an essential role in myocardial I/R injury [15, 16]. Autophagy is significantly activated during an I/R episode and likely contributes to cell death by excessively degrading essential proteins and organelles [2]. In addition, inhibition of excessive autophagy protects myocardial cells from I/R injury [3]. Therefore, we hypothesized that LncRNAHRIM similarly regulates autophagic activity. As shown in Fig. 3C and 3D, LncRNA-HRIM expression was decreased using siRNA-1. We observed that the LC3B-II/LC3B-I ratio, which indicates the level of autophagy, was significantly increased in the H/R group compared with the control group. Meanwhile, the LC3B-II/LC3B-I ratio and the total amount of LC3B-II and LC3B-I were markedly decreased in the siRNA-LncRNA-HRIM group compared to the H/R group. Moreover, the number of autophagosomes detected by electron microscopy displayed the same tendency (Fig. 4A). LC3 was tagged and tracked by expression of GFP and mRFP in the tandem GFP-RFP-LC33 adenovirus. The increase or decrease in autophagosome formation is accompanied by the increased or decreased utilization of GFP-RFP-LC33, which displays red and green fluorescence under microscopy. Under reduced $\mathrm{pH}$, GFP degrades from the tandem protein when the autophagosome fuses with a lysosome to form autolysosomes, whereas RFP-LC3 expression is maintained as puncta. Hence, the process from autophagosomes to autolysosomes can be monitored by changes in fluorescence [17]. Both fluorescent proteins were observed after successful infection with GFP-RFP-LC3, and colocalized to resemble yellow puncta (Fig. 4B). In addition to LC3 accumulation, fewer yellow puncta were detected in H9c2 myocytes transfected with si-LncRNA-HRIM than in

Fig. 3. Knockdown of IncRNA-HRIM decreased the ratio of LC3B-I/LC3BII and increased cell viability of $\mathrm{H} 9 \mathrm{c} 2$ myocytes. (A) H9c2 myocytes were transfected with three different siRNAs (designated as siRNA-1, siRNA-2 and siRNA-3) against IncRNA-HRIM. LncRNA-HRIM expression was detected by q-PCR. GAPDH was designed as an endogenous reference gene. (B) Cell viability of $\mathrm{H} 9 \mathrm{c} 2$ myocytes in the control, $\mathrm{H} / \mathrm{R}, \mathrm{H} / \mathrm{R}+\mathrm{NC}$, and $\mathrm{H} /$ $\mathrm{R}+$ siRNA-1 groups. (C) Western blot analysis of LC3-I, LC3-II, and GAPDH in H9c2 myocytes. (D) Quantification of panel A by densitometry. GAPDH was used as a loading control. Data (mean \pm SD) were obtained from three independent experiments. ${ }^{*} \mathrm{P}<0.01$ compared with the control group, \# $\mathrm{P}<0.01$ compared with the H/R group.

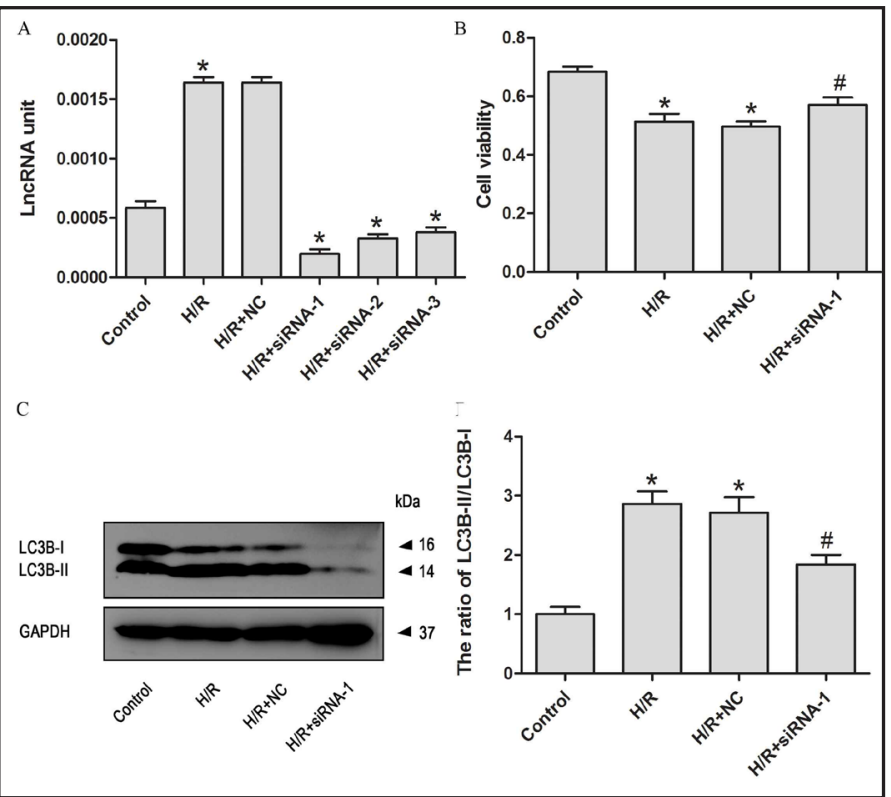


Fig. 4. Downregulation of IncRNAHRIM decreased autolysosome formation in $\mathrm{H} 9 \mathrm{c} 2$ myocytes under H/R compared with normal oxygen treatment. (A) Representative TEM images of intracellular ultrastructures of H9c2 myocytes. Four groups of cells were visualized, including the control, $\mathrm{H} / \mathrm{R}, \mathrm{H} / \mathrm{R}+\mathrm{NC}$, and $\mathrm{H} / \mathrm{R}+\mathrm{siRNA}-1$ groups. Pictures in the left and right columns were taken under 4,200X and 13,500X magnifications, respectively. The arrow designates an autophagosome. (B) Representative images of intracellular autolysosome flux detected by infecting GFP-RFP-LC3 adenovirus in $\mathrm{H} 9 \mathrm{c} 2$ myocytes subjected to $H / R$. The control, $H / R, H /$ $\mathrm{R}+\mathrm{NC}$, and $\mathrm{H} / \mathrm{R}+\mathrm{siRNA}-1$ groups were visualized. Pictures in the right column are a merge from

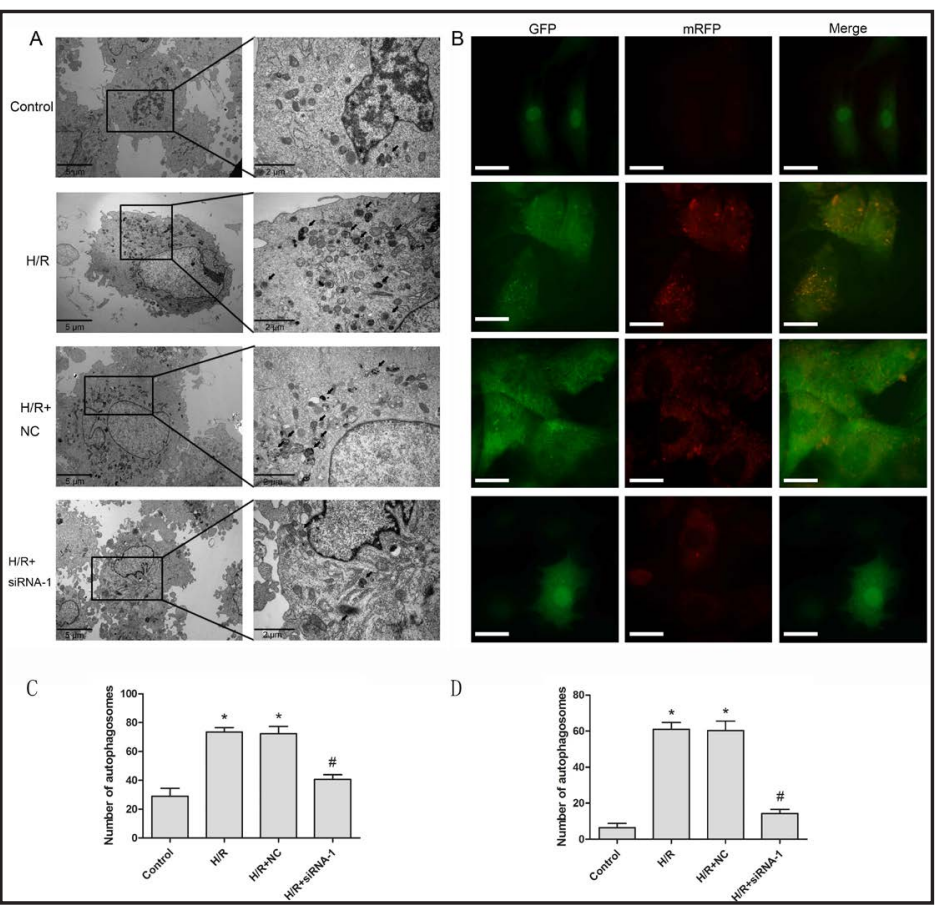
the first two columns. The yellow puncta represent autolysosome formation. Magnification, 200X. (C) Quantification of the average number of autophagosomes in each cell by TEM. (D) Quantification of the average autolysosome flux. Data (mean \pm SD) were obtained from three independent experiments. ${ }^{*} \mathrm{P}<0.01$ compared with the control group, \# $\mathrm{P}<0.01$ compared with the H/R group.

the H/R group without si-LncRNA-HRIM. These results further confirmed that depletion of LncRNA-HRIM expression inhibited autolysosome formation, indicating that LncRNA-HRIM mediated autophagy flux in H9c2 myocytes.

\section{Discussion}

In this study, we observed that LncRNA expression profiles of H9c2 myocytes changed during the H/R process. We selected 8 LncRNAs that may play critical roles in H/R injury. The differential expression levels of these 8 LncRNAs were validated in vitro and ex vivo by qPCR, which confirmed the microarray results. We focused on the function of LncRNA-HRIM (E230034005Rik), and demonstrated that inhibition of LncRNA-HRIM expression markedly enhanced myocardial cell activity via reduction of excessive autophagy during H/R. This result suggested that LncRNA-HRIM plays a detrimental role in regulation of H9c2 myocytes survival during the $\mathrm{H} / \mathrm{R}$ period.

Emerging evidence shows the critical roles of LncRNAs in the initiation and progression of cardiovascular diseases [18, 19]. Mitochondrial LncRNA uc022bqs.1 (LIPCAR) is downregulated during the early stages of MI but is upregulated during the later stages, suggesting that LIPCAR is a novel biomarker of cardiac remodeling and may predict death in patients with heart failure [20]. A recent study [21] showed that compared with healthy volunteers, MI patients have higher levels of hypoxia-inducible factor $1 \mathrm{~A}$ antisense RNA 2, KQT-like subfamily, member 1 opposite strand/antisense transcript 1 (KCNQ10T1), and metastasis-associated lung adenocarcinoma transcript 1 (MLAT1), as well as lower levels of cyclin-dependent kinase inhibitor 2B antisense RNA 1 (ANRIL). In addition, ANRIL, KCNQ10T1, and MLAT1 levels are lower in patients with ST-segment-elevation MI than in patients with non-ST-segment-elevation MI. Moreover, aberrant LncRNA expression (e.g.,

\section{KARGER}


Mhrt) is associated with the pathogenesis of ischemic heart failure [9]. These data indicate that LncRNAs are involved in the myocardial ischemia process. In the current study, we found that 309 LncRNAs were significantly upregulated and 488 LncRNAs were downregulated in H9c2 myocytes subjected to H/R. This finding suggested that LncRNAs may plays a role in heart damage. However, it was difficult to screen all differentially expressed LncRNAs for subsequent research based on the large chip data. We selected 8 LncRNAs to validate for the following reasons: (i) The LncRNAs were significantly upregulated or downregulatied in the H/R or I/R group compared with the control group, (ii) Several LncRNAs were validated LncRNAs that had the published sequences, and (iii) the contiguous coding-gene and coexpression of these 8 LncRNAs were related to autophagy or apoptosis, which play important roles in I/R injury.

The E230034005Rik gene, is a long non-coding RNA that is $1470 \mathrm{bp}$ in size. E230034005Rik is located on 20p12 and includes three exons. We selected LncRNAHRIM (E230034005Rik) for functional studies for the following reasons: (i) LncRNA E230034005Rik was significantly upregulated in the H/R or I/R group compared with the control group, (ii) E230034005Rik is a validated LncRNA and not a pseudogene, (iii) Clic1, the contiguous gene of E230034005Rik, is related to autophagy, which plays an important role in I/R injury and (iv) LncRNA-HRIM was co-expressed with Zdhhc7, Ptgis, Krt23 and Phactr1 mRNAs, which may be linked to the regulation of hypoxia/reoxygenation (H/R) injury in myocytes. Depletion of LncRNA-HRIM with siRNA significantly increased the viability of myocardial cells when myocytes were subjected to $H / R$. These observations suggested the potential adverse role of LncRNA-HRIM in H9c2 myocytes during H/R.

Most research efforts focus on differential LncRNA expression during the disease process $[22,23]$. However, the real mechanism or function of LncRNAs has not been completely clarified. For the current study, we selected LncRNA-HRIM (E230034005Rik) for preliminary functional studies.

Autophagy is a major pathway that responds to various extracellular and intracellular stimuli, such as nutrient deprivation, bacterial infection, and therapeutic treatment [24-27]. Autophagy is a dynamic biological process involved in membrane formation, autophagosome formation, autophagolysosome fusion, and autophagosome degradation, which involve signal transduction, RNA transcription, and protein synthesis. Appropriate autophagy levels are conducive to the survival of myocardial cells subjected to different stress or damage [28]. However, the excessive activation of autophagy degrades some important cell structures, which eventually leads to cell death [29]. Our previous experiments demonstrated that autophagy adversely affects myocardial cells during the H/R period $[3,4]$ and that inhibition of excessive autophagy increases the viability of myocytes. These results indicated that autophagy regulation is an effective strategy for treating H/R injury.

Several studies support the fact that LncRNAs participate in the regulation of autophagy [30-32]. LncRNA-MEG3 (Maternally Expressed Gene 3) is expressed at a low level in bladder cancer tissues compared with normal controls. Meanwhile, the downregulation of MEG3 increases cell proliferation in bladder cancer by activating autophagy [33]. Lnc-RNA-TGFB2OT1, as a competing endogenous RNA, regulates autophagy and inflammation in vascular endothelial cells by binding to MIR3960, MIR4488, and MIR4459[34]. In addition, LncRNAAPF (autophagy promoting factor) regulates miR-188-3p and affects autophagic cell death and myocardial infarction [35]. Therefore, the role of LncRNAs in the regulation of autophagy has attracted the attention of researchers. Previous studies have shown that ZDHHC7 [36, 37], Ptgis [38], and Krt23 [39] are involved in regulating autophagy or apoptosis. We observed that these proteins are co-expressed with LncRNA-HRIM by qPCR (see online suppl. material, Suppl. Fig. S2), which suggests that LncRNA-HRIM may be involved in regulation of the autophagic process. However, the target genes of LncRNA-HRIM, and the detailed mechanism need further research to confirm. 


\section{Cellular Physiology Cell Physiol Biochem 2018;46:1341-1351 \begin{tabular}{l|l} 
and Biochemistry Published onlIne: April 23, 2018 & $\begin{array}{l}\text { (c) } 2018 \text { The Author(s). Published by S. Karger AG, Basel } \\
\text { www.karger.com/cpb }\end{array}$
\end{tabular}}

Huang et al.: LncRNA-HRIM Regulated Autophagy

\section{Conclusion}

The LncRNA profiles of H9c2 myocytes changed extensively during the H/R period. Moreover, inhibition of LncRNA-HRIM increased cell viability partly by inhibiting autophagic activity, This finding suggests that the regulation of LncRNA-HRIM is a promising target for mitigating I/R injury.

\section{Acknowledgements}

This study was supported by the National Natural Science Foundation of China (Grant No. 81670227), the Health Bureau of Zhejiang Province (Grant No. 2016DTA004), the Natural Science Foundation of Zhejiang Province (LY13H020005 and LQ15H020005) and Wenzhou Science and Technology Bureau (Grant No: Y20170045).

\section{Disclosure Statement}

No conflict of interests exists.

\section{References}

1 Hausenloy DJ, Yellon DM: Myocardial ischemia-reperfusion injury: A neglected therapeutic target. J Clin Invest 2013;123:92-100.

2 Gustafsson AB, Gottlieb RA: Autophagy in ischemic heart disease. Circ Res 2009;104:150-158.

-3 Huang Z, Han Z, Ye B, Dai Z, Shan P, Lu Z, Dai K, Wang C, Huang W: Berberine alleviates cardiac ischemia/ reperfusion injury by inhibiting excessive autophagy in cardiomyocytes. Eur J Pharmacol 2015;762:1-10.

4 Huang Z, Ye B, Dai Z, Wu X, Lu Z, Shan P, Huang W: Curcumin inhibits autophagy and apoptosis in hypoxia/ reoxygenation-induced myocytes. Mol Med Rep 2015;11:4678-4684.

-5 Su YJ, Yu J, Huang YQ Yang J: Circulating long noncoding rna as a potential target for prostate cancer. Int J Mol Sci 2015;16:13322-13338.

6 Sigdel KR, Cheng A, Wang Y, Duan L, Zhang Y: The emerging functions of long noncoding rna in immune cells: Autoimmune diseases. J Immunol Res 2015;2015:848790.

-7 Marques-Rocha JL, Samblas M, Milagro FI, Bressan J, Martinez JA, Marti A: Noncoding rnas, cytokines, and inflammation-related diseases. FASEB J 2015;29:3595-3611.

-8 Archer K, Broskova Z, Bayoumi AS, Teoh JP, Davila A, Tang Y, Su H, Kim IM: Long non-coding rnas as master regulators in cardiovascular diseases. Int J Mol Sci 2015;16:23651-23667.

9 Wu C, Arora P: Long noncoding mhrt rna: Molecular crowbar unravel insights into heart failure treatment. Circ Cardiovasc Genet 2015;8:213-215.

10 Klawitter PF, Murray HN, Clanton TL, Angelos MG: Reactive oxygen species generated during myocardial ischemia enable energetic recovery during reperfusion. Am J Physiol Heart Circ Physiol 2002;283:H16561661.

-11 Badavi M, Sadeghi N, Dianat M, Samarbafzadeh A: Effects of gallic acid and cyclosporine a on antioxidant capacity and cardiac markers of rat isolated heart after ischemia/reperfusion. Iran Red Crescent Med J 2014;16:e16424.

12 Yang F, Zhang L, Huo XS, Yuan JH, Xu D, Yuan SX, Zhu N, Zhou WP, Yang GS, Wang YZ, Shang JL, Gao CF, Zhang FR, Wang F, Sun SH: Long noncoding rna high expression in hepatocellular carcinoma facilitates tumor growth through enhancer of zeste homolog 2 in humans. Hepatology 2011;54:1679-1689.

13 Huang Z, Wang L, Meng S, Wang Y, Chen T, Wang C: Berberine reduces both mmp-9 and emmprin expression through prevention of p38 pathway activation in pma-induced macrophages. Int J Cardiol 2011;146:153-158.

-14 Setti M, Osti D, Richichi C, Ortensi B, Del Bene M, Fornasari L, Beznoussenko G, Mironov A, Rappa G, Cuomo A, Faretta M, Bonaldi T, Lorico A, Pelicci G: Extracellular vesicle-mediated transfer of clic1 protein is a novel mechanism for the regulation of glioblastoma growth. Oncotarget 2015;6:31413-31427.

15 Sadoshima J: The role of autophagy during ischemia/reperfusion. Autophagy 2008;4:402-403.

16 Sciarretta S, Hariharan N, Monden Y, Zablocki D, Sadoshima J: Is autophagy in response to ischemia and reperfusion protective or detrimental for the heart? Pediatr Cardiol 2011;32:275-281. 


\section{Cellular Physiology Cell Physiol Biochem 2018;46:1341-1351 \begin{tabular}{l|l} 
and Biochemistry Published onIIne: April 23, 2018 & $\begin{array}{l}\text { (c) } 2018 \text { The Author(s). Published by S. Karger AG, Basel } \\
\text { www.karger.com/cpb }\end{array}$
\end{tabular}

17 Wang X, Liu J, Zhen J, Zhang C, Wan Q Liu G, Wei X, Zhang Y, Wang Z, Han H, Xu H, Bao C, Song Z, Zhang X, Li N, Yi F: Histone deacetylase 4 selectively contributes to podocyte injury in diabetic nephropathy. Kidney Int 2014;86:712-725.

18 Ma Y, Ma W, Huang L, Feng D, Cai B: Long non-coding rnas, a new important regulator of cardiovascular physiology and pathology. Int J Cardiol 2015;188:105-110.

19 Meganathan K, Sotiriadou I, Natarajan K, Hescheler J, Sachinidis A: Signaling molecules, transcription growth factors and other regulators revealed from in-vivo and in-vitro models for the regulation of cardiac development. Int J Cardiol 2015;183:117-128.

20 Kumarswamy R, Bauters C, Volkmann I, Maury F, Fetisch J, Holzmann A, Lemesle G, de Groote P, Pinet F, Thum T: Circulating long noncoding rna, lipcar, predicts survival in patients with heart failure. Circ Res 2014;114:1569-1575.

-21 Vausort M, Wagner DR, Devaux Y: Long noncoding rnas in patients with acute myocardial infarction. Circ Res 2014;115:668-677.

-22 Liu W, Pu Y, Wang C, Gu C, Zhang X: Expression profiles of long noncoding rnas in cardiac stem cells under hyperglycemic conditions. Int J Cardiol 2016;222:933-939.

23 Zhang M, Gu H, Xu W, Zhou X: Down-regulation of LncRNA malat1 reduces cardiomyocyte apoptosis and improves left ventricular function in diabetic rats. Int J Cardiol 2016;203:214-216.

24 Klionsky DJ: Autophagy: From phenomenology to molecular understanding in less than a decade. Nat Rev Mol Cell Biol 2007;8:931-937.

25 Maiuri MC, Zalckvar E, Kimchi A, Kroemer G: Self-eating and self-killing: Crosstalk between autophagy and apoptosis. Nat Rev Mol Cell Biol 2007;8:741-752.

26 Mizushima N, Klionsky DJ: Protein turnover via autophagy: Implications for metabolism. Annu Rev Nutr 2007;27:19-40.

27 Rubinsztein DC, Gestwicki JE, Murphy LO, Klionsky DJ: Potential therapeutic applications of autophagy. Nat Rev Drug Discov 2007;6:304-312.

28 Tai S, Hu XQ, Peng DQ Zhou SH, Zheng XL: The roles of autophagy in vascular smooth muscle cells. Int J Cardiol 2016;211:1-6.

29 Degterev A, Yuan J: Expansion and evolution of cell death programmes. Nat Rev Mol Cell Biol 2008;9:378390.

-30 Zhao Y, Guo Q Chen J, Hu J, Wang S, Sun Y: Role of long non-coding rna hulc in cell proliferation, apoptosis and tumor metastasis of gastric cancer: A clinical and in vitro investigation. Oncol Rep 2014;31:358-364.

-31 Pawar K, Hanisch C, Palma Vera SE, Einspanier R, Sharbati S: Down regulated LncRNA meg3 eliminates mycobacteria in macrophages via autophagy. Sci Rep 2016;6:19416.

-32 Ge D, Han L, Huang S, Peng N, Wang P, Jiang Z, Zhao J, Su L, Zhang S, Zhang Y, Kung H, Zhao B, Miao J: Identification of a novel mtor activator and discovery of a competing endogenous rna regulating autophagy in vascular endothelial cells. Autophagy 2014;10:957-971.

33 Ying L, Huang Y, Chen H, Wang Y, Xia L, Chen Y, Liu Y, Qiu F: Downregulated meg3 activates autophagy and increases cell proliferation in bladder cancer. Mol Biosyst 2013;9:407-411.

-34 Huang S, Lu W, Ge D, Meng N, Li Y, Su L, Zhang S, Zhang Y, Zhao B, Miao J: A new microrna signal pathway regulated by long noncoding rna tgfb2-ot1 in autophagy and inflammation of vascular endothelial cells. Autophagy 2015;11:2172-2183.

-35 Wang K, Liu CY, Zhou LY, Wang JX, Wang M, Zhao B, Zhao WK, Xu SJ, Fan LH, Zhang XJ, Feng C, Wang CQ Zhao YF, Li PF: Apf LncRNA regulates autophagy and myocardial infarction by targeting mir-188-3p. Nat Commun 2015;6:6779.

-36 Younce CW, Wang K, Kolattukudy PE: Hyperglycaemia-induced cardiomyocyte death is mediated via mcp-1 production and induction of a novel zinc-finger protein mcpip. Cardiovasc Res 2010;87:665-674.

37 Liu ST, Chang YL, Wang WM, Chung MH, Lin WS, Chou WY, Huang SM: A non-covalent interaction between small ubiquitin-like modifier-1 and zac1 regulates zac1 cellular functions. Int J Biochem Cell Biol 2012;44:547-555.

-38 Nakayama T: Genetic polymorphisms of prostacyclin synthase gene and cardiovascular disease. Int Angiol 2010;29:33-42.

-39 Birkenkamp-Demtroder K, Hahn SA, Mansilla F, Thorsen K, Maghnouj A, Christensen R, Oster B, Orntoft TF: Keratin23 (krt23) knockdown decreases proliferation and affects the DNA damage response of colon cancer cells. PLoS One 2013;8:e73593. 